\title{
Implications of measurement of eye fixations for a psychophysics of form perception*
}

\author{
MARY ANNE BAKER \\ Indiana University Southeast, Jeffersonville, Indiana 47130 \\ and \\ MICHEL LOEB \\ University of Louisville, Louisville, Kentucky 40208
}

\begin{abstract}
It was suggested that insights into feature analysis of processes involved in form identification might be gained from an analysis of eye movements made by Ss as they identified patterns. Fixations were measured during identifications of histoforms, polygons, and Vargus 10 figures. Eye fixations were measured, and Ss rated sections of the figures in terms of their importance. Eye fixations were measured in terms of number of changes and duration of fixations. The number of changes in fixation were found to reflect only individual differences. Duration of fixation was found to vary significantly with location within figures, with fixations being longest where changes of contour occurred. There was also a tendency to look longer at the top of polygons and Vargus 10 figures and at the center of histoforms. Ratings of importance were highest for sections of figures fixated for longer duration-generally areas in which changes of contour were present.
\end{abstract}

A number of perceptual theorists-Bruner, Hebb, J. J. Gibson, and E. J. Gibson, among others-have indicated that perceptions of complex patterns are built up from sensory representations of simple elements. It has been shown that, when confronted with patterns, especially large ones, Ss tend to fixate successively on more than one element, in a systematic fashion related to the individual stimulus and to the elements within it that contain the most information (Mackworth \& Morandi, 1967; Gould \& Dill, 1969). The studies cited, unlike the one to be described, employ either complex realistic stimuli (Mackworth \& Morandi, 1967) or stimulus patterns with many forms, rather than a simpler abstract figure consisting of one form.

Research on form perception in the past has involved various techniques for generating such forms. Attneave and Arnoult (1956) evolved a number of these, and they have pointed out that most form-perception studies suffer because of a lack of methodology to specify pattern populations and similarity among them. Fitts and others (e.g., Fitts \& Leonard, 1957; Baker \& Alluisi, 1962) evolved a different technique to construct histogram-like figures ("metric histoforms") that allows

*This research, which partially fulfilled the requirements for the $\mathrm{PhD}$ degree at the University of Louisville for the senior author, was aided by a contract between the U.S. Army Medical Research and Development Command, Office of the Surgeon General, and the University of Louisville, "Vigilance: Factors Influencing Detection and Monitoring Performance," and by U.S. Army Themis Contract A-HL19-69-C-0009, "Studies of Performance Assessment and Enhancement." The authors are indebted to E. A. Alluisi. G. S. Harker. T. Lawwill, and J. B. Thurmond for their advice and to Cardi Fleck for technical assistance. for specification of populations of forms in information-theory terms, which involves fitting columns of various lengths in a matrix. It has been shown that with figures constructed thus, average identification time for a form increases as the uncertainty or information content of a figure increases (Baker \& Alluisi, 1962; Alluisi \& Hall, 1965). Thurmond and Alluisi (1967) devised a way of constructing polygons, resembling those of Attneave and Arnoult, utilizing a polar matrix in a manner analogous to that used with Cartesian coordinates to generate the metric histoforms. In this experiment, Thurmond and Alluisi reported that performance was similar for the polygons and histoforms, but in a later one (Thurmond \& Hancock, 1969) it was reported that RT was less for polygons.

Most experiments have employed rectilinear rather than curvilinear forms. It has been reported that when curvilinear forms are transformed to rectilinear equivalents, they are perceptually more similar to other rectilinear forms (Edelman, 1960). Evans and others at Texas Christian University have generated patterns with curvilinear elements, using the "Vargus 10" computer programs. These are closed-line unfamiliar figures containing loops, curves. angles, and straight lines (Arnoult, 1968). Ss viewing these reported that corners were picked as features most relevant for identification (Hastings \& Evans. 1970).

Brown and Owen (1967) and Mavrides and Brown (1969) have attempted to specify the space in which Arnoult and Attneave's figures (Type I-see Attneave \& Arnoult. 1956) may be specified. In their method. figures with the same number of sides were considered to belong to the same population. In Brown and Owen's 
(1967) experiment, five populations were specified, 164 physical measurements were made, and the data were subjected to factor analytic technique. Five factors were identified (compactness, jaggedness, area skewness on $X$ and on $Y$ axes, rotation), which seemed to predict judgments of similarity (Aiken \& Brown, 1969) and latency (Brown \& LoSasso, 1967).

In spite of the diversity of research thus far employed, it has been concluded that only a beginning has been made on the development of a psychophysics of form perception (Michels \& Zusne, 1965; Brown \& Owen, 1967). It may be that there has been too much concentration on physical specification and too little on the relationship of behavioral measures relevant to physical components. It is possible, as was previously indicated, that a study of eye fixations may indicate to which stimulus elements Ss are attending in different stimulus patterns. In the present study, three different kinds of patterns were employed and Ss' eye fixations on each of these patterns were analyzed.

\section{METHOD}

Each $\mathbf{S}$ served in three experimental sessions and performed two different tasks. In Task I, eye fixations were recorded as Ss viewed three different types of forms. In Task II, Ss rated sections of the forms they had seen in Task I for informativeness.

\section{Subjects}

Nine freshmen at Indiana University Southeast, Jeffersonville, served as Ss. Each S served in three sessions; each session was separated from the other sessions by at least 1 day.

\section{Task I}

\section{Apparatus}

The Polymetric Eye Movement Recorder, wide angle, Model V-1166 version of the Mackworth eye camera (Mackworth, 1968) was used to record locus of fixation during inspection of the stimuli. In this technique, an image of the right eye and a reflection of the stimulus object reflected on the eye are recorded by a Beaulieu R16 camera on $16-\mathrm{mm}$ reversal film. After the fixation point of the pupil is located, the locus of fixation within the stimulus can be determined.

\section{Stimuli}

Ten different black silhouettes of each of three different types of forms were used as stimuli. Random $4 \times 4$ histoforms (Alluisi, 1960), random $4 \times 4$ polygons (Thurmond \& Alluisi, 1967), and Vargus 10 patterns (Evans \& Breckenridge, 1968) were employed. Samples of the types of forms are seen in Fig. 1. Each form was cut from a 6-in. square of black plastic material and placed on a $9 \times 11$ in. piece of clear plastic. The stimuli were presented 14 in. from the S's eye.

\section{Procedure}

In each session, Ss were seated in front of the apparatus and were instructed to hold their heads steady in the viewing porthole. They were told that they would be asked to identify forms and that a camera would be taking pictures of their eyes during the identification task.
Patterns were presented sequentially in groups of three, and Ss were asked to judge if the second pattern, the third, or neither of these patterns was the same as the first or target pattern. The actual measurement taken was of the eye-fixation patterns of the Ss. Each pattern was presented for $3 \mathrm{sec}$.

\section{Experimental Design}

There were three experimental sessions for each S. During any given session, the $\mathrm{S}$ was presented with 20 sequential target-identification problems, in which three patterns were presented sequentially, as indicated above. All problems in that session contained the same type of histoform, polygon, or Vargus 10 patterns.

For each type of form, 10 problems were constructed in which each of the 10 stimulus figures served as a target. The correct responses were counterbalanced so that in 3 of the 10 problems, the second pattern was the same as the first; in 3 , the third was the same; and in 3 , neither was the same as the target. In the 10th problem, the second was the same for one group of forms, the third in another, and neither was in a third group.

The order of presentation of the 10 problems was randomized twice for each $\mathrm{S}$. Each $\mathrm{S}$ was presented each problem twice to insure that there would be usable data for each problem for each $S$. The order of presentation of the types of forms was counterbalanced, with three Ss beginning with each of the types of forms.

\section{Scoring}

The film was processed and scored frame by frame in a viewer-editor. Using the data from the instructed fixations, the location on the eye for the point of foveal focus was determined and recorded on a piece of clear plastic, on which were also drawn the outside edge of the iris and the pupil. Both of the edges were included for points of reference, since the size of the pupil was not stable. The size of the pupil changes as light adaptation and accommodation occur during a stimulus presentation.

If, on a given problem, the eye was closed or the image blurred because the eye moved while the frame was being shot, or if the $S$ looked out of the field of the stimulus, then no attempt at scoring was made, and the second occurrence of the same problem was used for scoring. This situation occurred rarely-only on about 15 of the 270 problems.

Each type of form was subdivided into nine sections (Fig. 1). Polygons and Vargus 10 patterns were divided into nine square sections. Histoforms were divided into nine vertical sections. The goal in making all the divisions was to have only one critical element in each section.

Two independent measures, the number of changes in fixation and the duration of fixations, were taken on the data and analyzed.

\section{Task II}

In Task II, Ss were asked to rate sections of the figures for importance in identifying the whole figure.

\section{Procedure}

At the end of each Task I session, the Ss were presented with a set of 10 cards. Each card contained one of the 10 figures that he had just been viewing in Task I. Each figure was divided into nine sections, as indicated in $F$ ig. 1 . The $S$ was asked to rate each section of the figure on a 5-point discrete scale according to how important he felt that section would be in identifying the whole figure if the figure were cut into pieces and he were to see only one section at a time. The scoring for each figure was on a 
different piece of paper. so that comparisons between ratings on different figures were discouraged.

Analysis

For each type of form, a Kruskal-Wallis one-way analysis of variance of ranks (Siegel, 1956) was performed on the rating for each section of each figure, with the scores summed across Ss.

\section{RESULTS}

\section{Analysis of Task I}

The eye-fixation data from each type of form (histoform, polygon, and Vargus 10 patterns) were recorded and analyzed separately. For each type of form, two independent measures were taken. For the first measure, the number of changes in fixation, a two-factor analysis of variance with more than one score per $S$ was performed. For the second measure, the total duration of eye fixations within each section of the form was recorded and a three-factor (Ss by Figure by Location Within the Figure, with more than one score per cell) analysis of variance was performed.

\section{Number of Fixations}

The results of the analyses of variance on all three types of patterns-polygons, histoforms, and Vargus 10 -indicated that for each the only significant factor was $S$ s $[F(8.90)=8.16, F(8,90)=2.87, F(8,90)=$ 3.06 , respectively: $p<.01$ in all three cases]

A visual comparison of the data, plotted in terms of the mean number of changes in fixation for each type of form for each $\mathrm{S}$. suggests considerable similarity in the fixation pattern for a $S$ across types of forms. A Kendall coefficient of concordance (Siegel, 1956) supports this conclusion $(\mathrm{W}=0.75, \mathrm{p}<.05)$.

Tests of the differences between the overall average mean for number of fixations for each type of form were also performed. The mean for polygons equaled 4.40 , the mean for histoforms equaled 6.22 , and the mean for Vargus 10 patterns equalled 6.93 . The results of a $t$ test of the difference between the means of the Vargus 10 patterns and the histoforms indicate no significant difference $(t=1.69, p<.10)$. The results of a $t$ test of the difference between the means of the Vargus 10 patterns and polygons and the difference between the histoform and polygon means are significant $(t=5.75$ and $t=3.79$, respectively; $p<.01)$. These differences suggest that the polygons are perceived as simpler figures than the histoform and Vargus 10 patterns, which could be considered not perceptually different in complexity.

\section{Duration of Fixation Data}

The results of the three analyses of variance-one for each type of form -.on the duration of fixation data are
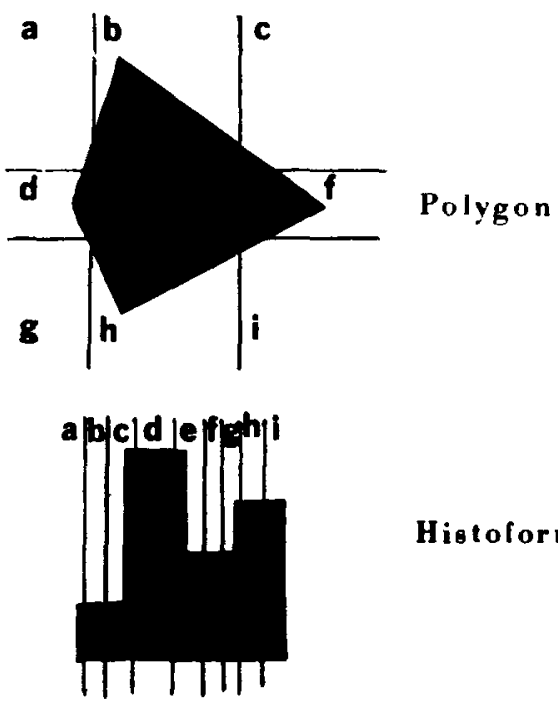

\section{Histolorm}

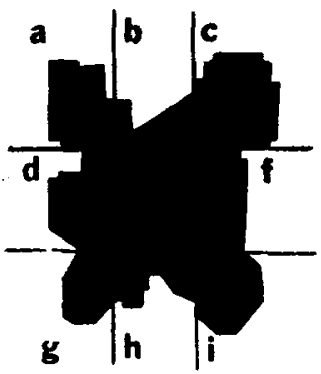

Vargus: 10

Fig. 1. Samples of the three types of forms used as stimuli with scoring grid superimposed.

similar to each other. For all three types of forms, a significant difference in location of fixations within a figure was found. No other main effects are significant. Two $S$ interactions (Ss by Location and Ss by Figure by Location) are significant for all three types of forms. (In this and subsequent analyses, significant $S$ interaction was, of course, employed as error terms in the appropriate manner.) The interaction of Location by Particular Figure Sampled is significant for both histoforms and polygons.

Polvgons. The results of the analysis of variance on the duration of fixations for polygons indicated that differences between particular figures sampled and between Ss were not significant. The only significant main effect was that due to location within the figure $[F(8.64)=18.50 . p<.01]$.

Figure 1 shows the manner in which all figures within this class of forms were subdivided into nine sections for purposes of scoring. as well as for use in Task II. Within this grid, one of the corners of the figure is always contained in Sections B. D. F, and H: Figure 2 shows that eye tixations were almost exclusively limited to these four sections. In wher words. Ss looked at comers 


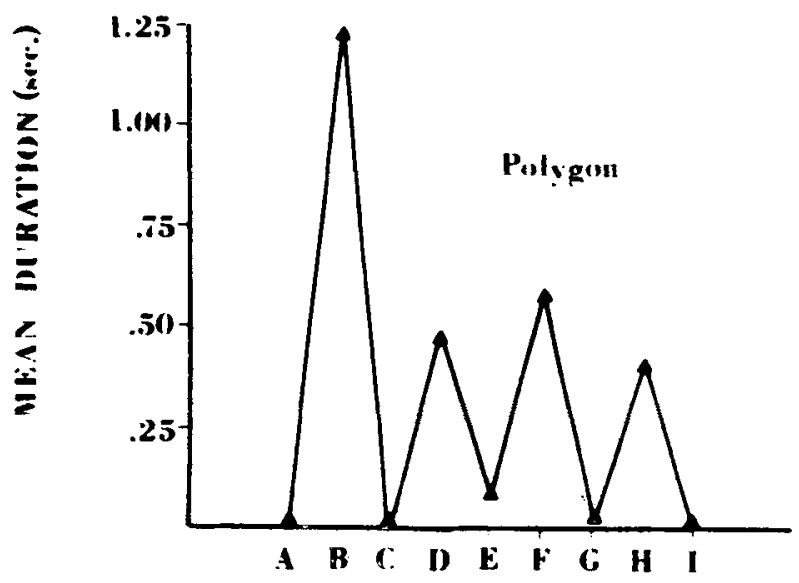

\section{I.OCATIOS A ITHIX HICI RE:}

Fig. 2. Mean duration of fixations (in seconds) for each location within polygons. Corners are located in Sections B, D, $F$, and $H$.

and not at sections that contained either short sections of straight lines or solid white or black areas.

A Newman-Keuls analysis (Winer, 1962) of these means shows that Sections A, C, E, G, H, and I do not differ significantly from each other; that Sections $D$ and $F$ do not differ significantly from each other but do differ from Sections $A, C, E, G, H$, and $I$; and that Section B differs significantly from all the other sections. These findings can be summarized by saying that Ss look at corners, the information-carrying sections of the figure, more than they look at the other sections, and further that Ss show a top to bottom preference.

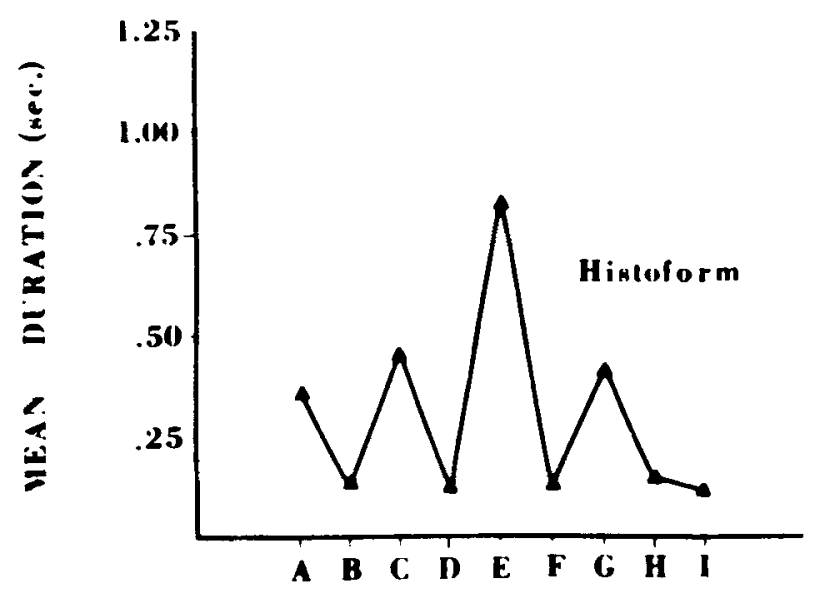

\section{LOCATION WITHIN FICURE}

Fig. 3. Mean duration of fixations (in seconds) for each location within histoforms. Changes in column heights occur in Sections A, C, E, G, and I.
Duration of fixations for the bottom corner does not differ significantly from the duration of fixations on noncorner sections.

The interaction of the Location Within the Figure by the Particular Figure Sampled from the class of forms. polygons, was also significant $[F(72.576)=2.37$. $p<.01]$. Because the interaction has 90 elements, the data were broken down into 10 subsections. such that for each of the 10 particular figures used in the experiment an analysis of the duration of fixation for each location within that figure could be carried out.

The pattern of fixation for each figure is similar to the average shown in Fig. 2. That is, during most of the duration of the presentation of the stimulus, Ss fixated on corners. However, the relative duration of fixations at the corners was not the same for all figures. The top corner (Section B) is always fixated for the longest duration. The duration of fixation for the other corners varies from figure to figure. The same general pattern emerges here as occurred in the analysis of the location main effect. The sections subdivide into two groups, with all the noncorner sections (A, C, E, G, and I) always having the five shortest durations of fixations and the four corners (Sections B, D, F, and H) always having the four longest durations of fixation. The significant interaction effect comes from the variation in the order of sections within each subgroup.

The $S$ by Location interaction $[F(64,810)=19.55$, $\mathrm{p}<.01]$ and the $S$ by Figure by Location interaction $[F(576,810)=1.30, p<.01]$ are also significant.

Histoforms. The results of the analysis of variance performed on the duration of fixations with histoforms indicate that differences between particular figures and between Ss are not significant. The only significant main effect is that due to variation in the duration of fixation for different figure locations $[F(8,64)=23.44, p<.01]$. The grid for dividing the figures into nine subsections can be seen in Fig. 1.

Since the information in histoforms is distributed along only one side-the top side in the present experiment-a grid that has nine vertical sections was chosen rather than the square grid used for the histoforms and polygons. When a nine-section vertical grid is applied to a $4 \times 4$ histoform, four sections occur in the middle of columns (labeled $B, D, F$, and $H$ ); five sections are located where changes in column height occur (labeled A, C, E, G, and I).

It can be seen in Fig. 3 that the greatest durations of fixation occur at the point that changes in column heights occur. Of the sections in which changes in column height occur, only Section I does not differ significantly in a Newman-Keuls analysis from Sections $B, D, F$, and $H$, which are situated in the middle of columns. Sections A, C, and G do not differ significantly from each other, but do differ significantly from Sections B, D, F, H, and I. Section E differs significantly from all the other sections. 
The interaction of Location Within a Figure by Particular Figure Sampled from the Population of Histoforms was also significant $[F(72,576)=4.48$, $p<.01]$. As in the previous analysis of the polygon data, the 90 -element interaction was broken down into 10 subsections to facilitate analysis of the duration of fixations for each of the 10 figures used in the experiment.

A comparison of the curves for the different figures shows considerable variation. The variation in the duration of fixation between different figures appears to be related to the location of changes in column height (the loci of information in Attneave's definition) in the particular figure. From looking at Fig. 3, it could be hypothesized that Ss have a real bias for fixating within Section E. A comparison of the duration of fixation data with the individual figures indicates that the duration of fixations is related to the locus of information within the figure, and not to position bias alone. In figures where no actual change in column height occurs at Section E, duration of fixation does not differ significantly from other nonchange sections of the figure.

When a mean for a particular section differs significantly from other means, it is accompanied by an actual change in column height within that section. For example, even though a change in column height could occur in Section $C$ of a figure, if no change does occur, the mean for that section does not differ significantly from the means for Sections H, D, I, F, and A (see Fig. 1).

In general, it can be said that if a change occurs in Section $\mathrm{E}$, the longest duration of the fixation will occur in that section. If the only change in column height within the figure occurs at Section E, essentially all the exposure time will be spent in fixating that section.

Vargus 10 patterns. The results of the analysis of variance performed on the duration of fixation data using Vargus 10 patterns as stimuli indicated, as in the case of the other two stimulus categories, that the only significant main effect was that due to the location of fixation within the figure $[F(8.64)=8.56, p<.01]$. The mean duration of fixation per location is shown in Fig. 4.

Visual analysis shows that Sections A. B, and C are fixated for greater duration than any of the other six sections. As can be seen from Fig. 1, Sections A, B, and $C$ contain the top of the figure. Sections A and C contain the top right and left corners. The bottom corners are contained in Sections $G$ and $I$ : and Sections $\mathrm{B}, \mathrm{D}, \mathrm{F}$, and $\mathrm{H}$ contain elements that connect the corners. There is a general tendency toward a reduction in the duration of fixation at each lower row of elements and a tendency to fixate longer in high-information areas (corners). This pattern of preference is illustrated by the fact that the score for Section B. a top connecting side. does not differ significantly from the scores for Sections

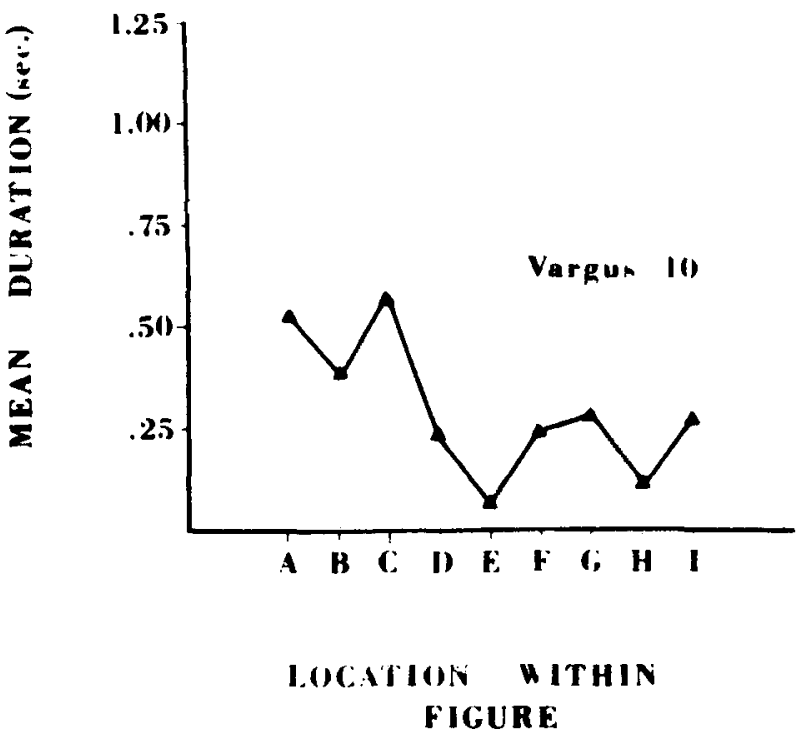

Fig. 4. Mean duration of fixations (in seconds) for each location within Vargus 10 figures. Corners are located in Sections A, C, G, and I.

$D$ and $F$, connecting side on the second row, or from the scores for Sections $G$ and $I$, corner section in the bottom row. Corners in the top row are fixated for the greatest duration, connecting sections in Rows 1 and 2 as well as corners in the third row are fixated for the next longest duration, and connecting sections in Row 3 and sections without edges are fixated for the shortest duration. Unlike the results of the analysis of the other two types of forms, the Duration of Fixation by Figure interaction is not significant $[F(72,576)=1.11, p>.10]$. The location preference appears to be independent of the figure presented. Vargus 10 patterns differ from the other types of figures. Information is always located at the same location and there is always information at a given section. Figures differ from each other in the particular configuration of rather complex line segments.

\section{Analysis of Task II}

Task II was performed by Ss at the end of each session. Ss were asked to rate each section of each of the figures that had been presented during that session.

For each type of form, a Kruskal-Wallis one-way analysis of variance of ranks (Siegel, 1956) was performed on the total score for each section, for each figure, summed across Ss. For polygons and Vargus 10 patterns, the results of this analysis are significant at the .001 level, for histoforms $p<.10$. These results indicate that the mean ranks for the nine sections do differ significantly. The greater variability of the histoform data is to be expected. since the high-information or corner areas do not always contain a change in line direction. This finding is consistent with the findings on the duration of fixation data.

The question is. do Ss rate highly those sections that 
they fixate for longer durations or do they use different criteria? The results of a Pearson product-moment correlation between fixation duration and rating for each section indicates that generally the sections rated as important are fixated for longer durations. The correlation for histoforms equals 0.52 ; for Vargus 10 patterns, 0.82 ; and for polygons, 0.59 .

While these correlations are substantial and impressive, it should not be inferred that the ratings and fixations provide precisely the same information; there are, in fact, important differences. It was noted in the previous sections that, while durations of eye fixations are greatest for sections of figures which would presumably carry the most information, there were additional systematic biases. Specifically, Ss fixated most of ten on the top corners of polygons, most often at the center and least often at the right column transition points of histoforms, and most often at the top corners of Vargus 10 figures. These biases are not reflected in the ratings; all corners of polygons, all transition points of histoforms, and all corners of Vargus 10 figures were rated approximately the same.

The results of the rating task can be summarized by saying that Ss judge certain sections as more important than others and that these judgments correspond significantly but imperfectly with the duration of fixation data.

\section{DISCUSSION}

The results of the present study (shown in Figs. 2, 3, and 4) confirm the previous findings that Ss do fixate for different durations at different locations (Mackworth \& Morandi, 1967; Gould \& Dill, 1969). They also extend the previous findings by indicating that Ss differentially fixate within the silhouettes of the type of figures that have often been used in form-perception studies.

It has been hypothesized that those elements of a figure for which more components of information must be encoded are fixated for longer durations. Gould and Dill (1969) suggest that the duration of fixation is determined by the time it takes to process the encoded information. The time is spent comparing the encoded elements with the standard prototype. The results of the duration of fixation data of the present study do not necessarily confirm the findings of Gould and Dill, neither do they support an alternative hypothesis that fixation time is a function of the number of elements to be encoded at a particular location.

Six of the Vargus 10 patterns used previously by Hastings and Evans (1970) were included in the present study. These six patterns contain some elements that occur more than once and at different locations within the figure. Hastings and Evans found that these elements were consistently judged either high or low in importance, regardless of the locations of the feature. The fixation data do not show this consistency, but rather they show great variation in duration of fixation from the same feature in different locations.

\section{Position Preference}

The location of fixation within figures appears to be related both to position preferences and to the location within the figure of the information as defined by Attneave (1957). In a previous study (Mackworth \& Morandi, 1967), checks for left-right fixation preferences were made by reversing the stimulus display; it was concluded that fixation locus was determined by the location of information-bearing sections of the stimulus, rather than a left-right preference. The data from the polygon and Vargus 10 forms tend to support this (see Figs 2 and 4). The histoform data, however, suggest that $S s$ tend not to look at the rightmost elements of the stimulus. This does not appear to represent a progressive reduction in duration of fixation, as one moves from left to right; rather, it appears that duration of fixation is essentially equal for all sections except the last column, where a sudden drop in duration of fixation occurs. This finding, while not directly supporting a left-right preference, does indicate that the extreme right part of a figure is, under some circumstances, fixated for shorter durations.

With polygons (see Fig. 2), Ss looked longest at Section $B$, the section in the most preferred top row containing a high-information location (a corner), and with Vargus 10 patterns (Fig. 4) Ss looked longest at Sections $A$ and $C$, the sections in the top row containing corners. There appears to be a position preference for the top of figures, but the particular location of the fixations is related to location of the corner sections. This is in keeping with other findings (Attneave, 1954; Mackworth \& Morandi, 1967; Michels \& Zusne, 1965) that the locations of fixations are correlated with the distribution of information within the stimulus.

The proportion of the total fixation time occurring within a section of a figure also appears to be correlated with the distribution of the information within that figure. When the polygons (figures that can be described in this experiment by specifying only the four corners) were used as stimuli, almost all of the fixation time was spent at these four locations (Fig. 2). When histoforms and Vargus 10 patterns (figures that have more complex perimeters) were used as stimuli, the relative difference in duration of fixation between corner and noncorner elements was not as great as that for polygons. This finding extends the previous findings by indicating that duration of fixations is distributed as the information is distributed. In other words, as the complexity of the figure increases, the distribution of fixations become more similar.

\section{Judged Importance of Features}

Mackworth and Morandi (1967) found that regions within the stimulus photograph containing contours with little change in direction were of ten judged higher in informativeness than the eye-fixation data would 
suggest. When the density of eye fixations, the stimulus figure, and the ratings were compared, they appeared to identify the regions of the figures with the highest interest or information value to the viewer.

The present findings also indicate that there are appreciable correlations between ratings of importance of sections of figures and durations of fixations of those sections. One might question, therefore, whether eye fixation measurement is worth the trouble, as it is easier and cheaper to obtain ratings. However, it will be recalled that there were biases such that different parts judged equally important were fixated for different durations. This is a reasonable finding, as ss can usually" identify figures in terms of some critical features without having to determine them all. Measurement of eye movements, then, does provide additional information in that it tells us what critical features are actually utilized by Ss in making their judgments. This would not be revealed by any of the rating techniques generally employed.

\section{Development of a Psychophysics of Form Perception}

For an adequate psychophysics of form perception to be established, a method of specifying the domain of the stimulus and that of the perception must be developed. Most past work attempting to specify the dimensions of form perception has attempted to specify the physical domain in terms of the dimensions used for geometrical descriptions of the forms-line, angle, area, measured distance, etc. (e.g., Attneave \& Arnoult, 1956; Brown \& Owen, 1967; Kaufman \& Richards, 1969)-or in terms of information, statistically defined (Fitts \& Leonard, 1957). Although the results have shown some correlation between some of the figural measures and performance, Michels and Zusne (1965) indicate that they have not led to a general psychophysics of form perception or to a generalized understanding of the problems involved. Indeed, Arnoult (1968) has identified as almost hopelessly complicated the task of limiting and at tacking the problem of form perception in these terms.

The population of forms approach, at least as it has been applied, appears to be of limited utility in developing a psychophysics of form perception. It cannot be assumed that the dimensions along which the forms were constructed are necessarily the only relevant ones. Moreover, these techniques do not attend to the effects of differences within the populations of figures as previous investigators have defined them (e.g., figures with the same statistical information content or figures with the same area or same number of angles, etc.). It has been shown. for example, that constrained and random figures with the same information content are not equally identifiable (Baker \& Alluisi. 1962: Thurmond. 1969).

The population of forms approach, then. while it may be a sound beginning for identifying relevant dimensions, must be supplemented by other techniques. An approach such, as the present one, in which the actual observing responses of the Ss are tabulated, would appear to be one such technique.

\section{CONCLUSIONS}

(1) Eye fixations are not random, but are related to the configuration that is fixated.

(2) The locations with the greatest durations of fixation tend to be high-information areas as defined by Attneave (1954). An improved method for identifying the high-interest areas of the stimuli might be developed with the use of eye-fixation data, so that the relevant physical space could be specified.

(3) Ss identify as important the areas upon which they fixate for the longest durations.

(4) Further attempts to develop a psychophysics of form perception might benefit from the findings about the location of eye fixations.

\section{REFERENCES}

Aiken, L. S., \& Brown, D. R. A spatial analysis of the discriminability of forms in noise. Perception \& Psychophysics, 1969, 5, 171-175.

Alluisi, E. A. On the use of information measures in studies of form perception. Perceptual \& Motor Skills, 1960, 11, 195-203.

Alluisi, E. A., \& Hall, T. J. Effects of a transphenomenal parameter on the visual perception of form. Psychonomic Science, 1965, 3, 543-544.

Arnoult. M. D. Psychophysical models for pattern perception. In, Pattern identification by man and machine. U.S. Army Technical Memorandum 17-68, 1968, 63-68.

Attneave, F. Some informational aspects of visual perception. Psychological Review, 1954, 61, 183-193.

Attneave, F. Transfer of experience with a class schema to identification-learning of patterns and shapes. Journal of Experimental Psychology, 1957, 54, 81-88.

Attneave, F., \& Arnoult, M. D. The quantitative study of shape and pattern perception. Psychological Bulletin, 1956, 53, $452-471$.

Baker, E. J., \& Alluisi, E. A. Information handling aspects of visual and auditory form perception. Journal of Engineering Psythology, 1962, 1,159-179.

Brown, D. R., \& LoSasso, J. S. Pattern degradation, discrimination difficulty, and quantified stimulus attributes. Psychonomic Science, 1967, 9, 351-352.

Brown, D. R., \& Owen, D. H. The metrics of visual form: Methodological dyspepsia. Psychological Bulletin, 1967. 68, 243-259.

Bruner, J. S. Going beyond the information given. In J. S. Bruner (Ed.). Contemporary approaches to cognition. Cambridge, Mass: Harvard University Press, 1957.

Edelman. S. K. Analysis of some stimulus factors involved in the associative response. Unpublished doctoral dissertation. Purdue University, 1960.

Evans. S. H.. \& Breckenridge, R. L. Modeling the pattern recognition environment. In. Partem identification by man and machine. U.S. Army Technical Memorandom 1768. 1968. 23-34.

Fitts. P. M.. \& Leonard. J. A. Stimulus correlates of visual pattern recognition-a probability approach. Office of Nival 
Research, Final Report of Contract Nons-495(02), October 1957.

Gibson, J. J., \& Gibson, E. J. Continuous perspective transformations and the perception of rigid motion. Journal of Experimental Psychology, 1957, 54, 129-138.

Gould, J. D., \& Dill, A. B. Eye-movement parameters and pattern discrimination. Perception \& Psychophysics, 1969, 6, 311-320.

Hastings, M. A., \& Evans, S. H. The selection of local features for pattern identification: An exploratory study. U.S. Army Technical Memorandum 5-70, 1970.

Hebb, D. O. The organization of behavior: A neuropsychological theory. New York: Wiley, 1949.

Kaufman, L., \& Richards, W. Spontaneous fixation tendencies for visual forms. Perception \& Psychophysics, 1969, 5, 85-88.

Mackworth, N. H. The side-angle reflection eye camera for visual choice and pupil size. Perception \& Psychophysics, 1968, 3, 32-34.

Mackworth, N. H., \& Morandi, A. J. The gaze selects informative details within pictures. Perception \& Psychophysics, 1967, 2, 547-552.

Mavrides, C. M., \& Brown, D. R. Discrimination and reproduction of patterns: Feature measures and constraint redundancy as predictors. Perception \& Psychophysics, 1969. 6, 226-228.

Michels, K. M., \& Zusne, L. Metrics of visual form. Psychological Bulletin, 1965, 63, 74-86.

Siegel, S. Nonparametric statistics for the behavioral sciences. New York: McGraw-Hill, 1956.

Thurmond, J. B. Effects of discriminability on the identification of different types of visual forms. Psychonomic Science, 1969, 15, 193-195.

Thurmond, J. B., \& Alluisi, E. A. An extension of the information-deductive analysis of form. Psychonomic Science, $1967,7,157-158$.

Thurmond, J. B., \& Hancock, J. B. Effects of figural complexity on the identification of different solid and outlined shapes. Psychonomic Science, 1969, 15, 315-317.

Winer, B. J. Statistical principles in experimental design. New York: McGraw-Hill, 1962.

(Received for publication June 12, 1972; revision received November 3,1972 .) 